\title{
3D Coupled Electro-mechanics for MEMS: Applications of CoSolve-EM
}

\author{
J.R. Gilbert ${ }^{*}, R$. Legtenberg ${ }^{\ddagger}$ and S.D. Senturia* \\ *Department of Electrical Engineering and Computer Science, M.I.T., Cambridge, MA. 02139 \\ ‡ MESA Research Institute, University of Twente, P.O. Box 217, 7500 AE Enschede, The Netherlands
}

\begin{abstract}
Micro-electro-mechanical systems (MEMS) are often designed on scales at which electrostatic forces are capable of moving or deforming the parts of the system. In this regime accurate prediction of device behavior may require $3 \mathrm{D}$ coupled simulations between the electrostatic and mechanical domains.

We have recently developed CoSolve-EM, a coupled solver for 3D quasi-static electro-mechanics. In this paper, we demonstrate the application of CoSolveEM to five classes of electro-mechanical problems that are often intractable to other techniques. These classes are: devices with electrostatic pull-in instabilities, devices in which precise deformations are required, devices driven by multiple conductors, capacitive sensors that make use of surface contact, and actuators that make use of surface contact.
\end{abstract}

\section{Introduction}

Previous approaches to this problem are: direct coupling of finite elements (FE) to a parallel plate approximation for the electrostatic force [1]; direct coupling of full FE solvers in both domains [2]; and external, boundary-based coupling between a mechanical FE solver and an electrostatic boundary element (BE) solver, using either relaxation or SNGCR techniques for convergence [3]. CoSolveEM implements the last of these approaches.

\section{General Coupled Electro-Mechanics}

When designing electro-mechanical actuators one typically applies a voltage to the undeformed device, as in Figure 1A. This induces charges on the surface of the conductors in the problem, and those charges induce surface normal pressures over the device. We refer to these pressures as the "electrostatic load" which may be calculated using Equation 1, in which $p$ is the normal outward pressure on a conductor, $\sigma$ is the charge density at that point, and $\varepsilon$ is the dielectric constant of the material outside the conductor.

The electrostatic load causes the device to deform. In general such deformation will lead to reorganization of all surface charges (and thus pressures) on the device, as illustrated in Figure 1B.

$p=\frac{\sigma^{2}}{2 \varepsilon}$

We are principally interested in that subset of MEMS devices in which this reorganization of charge is large enough to cause further deformation. We consider such devices to exhibit "coupled electromechanical behavior." In order to model them we must find self-consistent solutions in which the electrostatic loads are exactly balanced by the stresses of the solid deformation.

$\mathbf{A}$

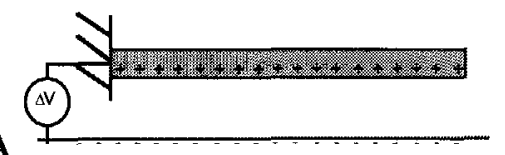

B

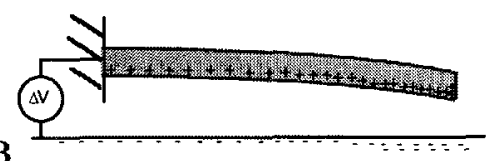

Figure 1 A schematic figure showing the reorganization of charge (and thus forces) accompanying the deformation in an electro-mechanical device.

\section{CoSolve-EM Software}

CoSolve -EM is a Coupled Solver for ElectroMechanics. It finds the self-consistent solution to the problem above using an external, boundarybased coupling between a mechanical FE solver and an electrostatic BE solver, implementing both relaxation and SNGCR techniques for convergence [3].

The two external solvers employed by CoSolve-EM are the commercial mechanical FE package ABAQUS [4], and a version of the electrostatic multipole-accelerated BE solver FASTCAP [5], CoSolve-EM also includes a 3D visualizer, Geomview, from the University of Minnesota [6]. The CoSolve core is written in $\mathrm{C}++$, with its user interface in Tk/tcl [7]. CoSolve-EM makes use of MemBase, our class library for doing general 
manipulations of meshed 3D models. MemBase gives CoSolve-EM the ability to break models up, add them together, extract surfaces, analyze structures for connectivity, and find all external and internal boundary regions. Using CoSolve-EM we can take one meshed model of a device, represented either in PATRAN Neutral File format or in I-DEAS Universal File format, and do all further electrostatic, mechanical or electro-mechanical modeling from the CoSolve-EM interface. CoSolve-EM is also useful for modeling general capacitance sensors, even those without significant electro-mechanical coupling (see the touch-down pressure sensor example below).

CoSolve-EM is built to use a heterogeneous cluster of workstations. In our current system the CoSolveEM core runs on a Sparc10 while the solvers ABAQUS and FASTCAP are run on a DEC 3000/800 (alpha cpu with OSF/1). We also have installed CoSolve-EM with ABAQUS and FASTCAP on a single Sparc10.

\section{The Examples}

We now present five examples of the use of CoSolveEM. In each case, only one meshed model is constructed, and only one run of CoSolve-EM is required. The five are: a torsion mirror, a deformable plate, a model of comb levitation, a touch-down capacitive pressure sensor, and a curved electrode actuator. We illustrate different aspects of electro-mechanical problems with each example, and also demonstrate different types of analysis that can be performed with CoSolve-EM.

CoSolve-EM implements two different algorithms for finding coupled electro-mechanical solutions, relaxation and SNGCR. Each has its merits. Relaxation is simple and often fast in practical examples, but can fail to converge for some problems. SNGCR is more reliable, but is more computationally expensive, and in our current implementation may not be used in problems containing surface contact.

Both the torsion mirror and the deformable plate are usually solvable with relaxation. The comb levitation problem generally requires (as do all levitation problems) the use of SNGCR. The touchdown pressure sensor, while not strictly a coupled problem, is easy to treat with CoSolve-EM. The last example, a curved electrode actuator, is a contact problem and thus must be handled by relaxation. However, the problem also contains some levitation, and would be more accurately solved by SNGCR.
This problem is discussed further in the section on the curved electrode actuator.

In Table 1 we summarize the sizes and some timing results for the five examples of this paper. We use the number of surface panels to indicate the size of each problem, and the time is the real elapsed time the user observes for one correct self-consistent solution. These times are not very well defined, because, even within one model, the difficulty in getting a converged solution actually can vary by a factor of 5 or more. We observe a great variation in elapsed time vs. voltage on a single problem if it has a pull-in instability or a touch-down. We are currently working on better techniques for benchmarking coupled electro-mechanics.

Table 1 Size is in number of surface panels. Time is in seconds for a single self-consistent solution at a voltage away from pull-in. These numbers are for our installation using a Sparc 10 for CoSolve-EM and a DEC 3000/800 for ABAQUS and FASTCAP.

\begin{tabular}{|l|c|c|}
\hline \multicolumn{1}{|c|}{ Example } & Size & Time \\
\hline Torsion Mirror & 2830 & 1750 \\
\hline Deformable Plate & 1580 & 800 \\
\hline Comb Levitation & 1837 & 1500 \\
\hline Touch-Down P Sensor & 3728 & $600^{*}$ \\
\hline Curved Electrode Actuator & 4720 & 2700 \\
\hline
\end{tabular}

* The touch-down sensor is not a coupled problem, so this time is for a single deformation and capacitance extraction.

\section{Torsion Mirror}

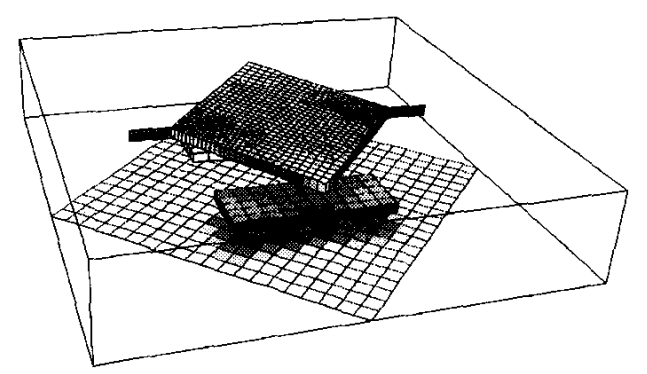

Figure 2 Torsion Mirror Plate, at 4.0 V. Mirror tip displacement is $0.65 \mu \mathrm{m}$, corresponding to a tilt angle of $1.3^{\circ}$. The figure is scaled by $5 x$ in $Z$ for visualization.

The torsion mirror is shown in Figure 2. The mirror plate is fixed at the ends of either arm, and able to twist about them. There are two electrodes below the 
mirror, and a ground plane below everything. A voltage is placed on one electrode (the forward one) and all other conductors are held at $0 \mathrm{~V}$.

One question to ask of this model is: What is the pull-in voltage of this device? [See [8] for more extensive discussions of pull-in in MEMS.] CoSolve-EM simulates pull-in by finding selfconsistent solutions at a sequence of applied voltages. This is shown in Figure 3. For this model pull-in occurs between $4.25 \mathrm{~V}$ and $4.5 \mathrm{~V}$. Figure 2 shows the deformation at $4.0 \mathrm{~V}$.

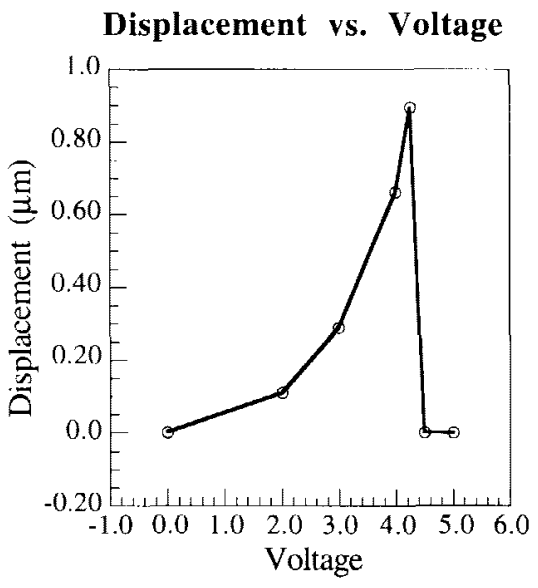

Figure 3 Graph of displacement vs. voltage for the tilting mirror plate. CoSolve-EM detects when the solution has gone beyond pull-in and sets the displacement to 0 , thus the last two points at $4.5 \mathrm{~V}$ and $5.0 \mathrm{~V}$ are shown as 0 displacement. Pull-in occurs between 4.25 and $4.5 \mathrm{~V}$.

\section{Controlled Deformation of Plates}

In applications such as adaptive optics, one may be interested in producing a controlled deformation in a reflective or refractive plate. To test the modeling of such a device, we built the following model: a square deformable plate $4000 \times 4000 \times 0.5 \mu \mathrm{m}$ suspended $5.5 \mu \mathrm{m}$ above a $5000 \times 5000 \mu \mathrm{m}$ ground plane. Between these plates three square $500 \times 500$ $\mu \mathrm{m}$ driving electrodes are positioned $3 \mu \mathrm{m}$ above the ground plane. The driving electrodes are placed to one side of the center of the system; they have centers at $\mathrm{x}=-1000$, and $\mathrm{y}=-1000,0$, and 1000 .

When we apply voltages to the electrodes we can control the deformation of the plate. Figure 4 shows two $X$ cross-sections of the deformed structure. Each cross-section is formed by taking the $\mathrm{x}-\mathrm{z}$ node coordinates of nodes within $100 \mu \mathrm{m}$ of $y=1000$ (the figure only displays nodes from the bottom face of the plate, the electrodes and the ground plane.) The circles are for the solution in which $0 \mathrm{~V}$ is applied to electrode $s 1$, and $30 \mathrm{~V}$ is applied to $s 2$, while the crosses are for the solution in which $30 \mathrm{~V}$ are applied to each of those electrodes, In both cases the plate, $s 3$ and the ground plane are all held at $0 \mathrm{~V}$.

\section{$\mathrm{X}$ cross-section}

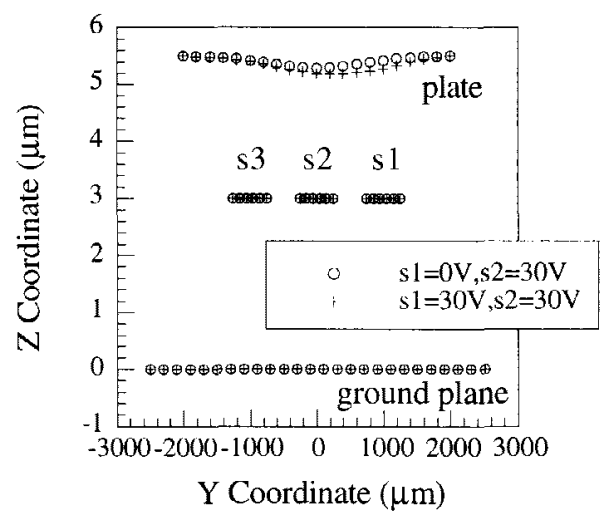

Figure $4 \quad X$ cross-sections at $x=-1000$, for two different electrostatic loads on $s 1$ and $s 2(s 3$, the plate and the ground plane are held at $0 \mathrm{~V}$.)

Figure 5 shows a magnified view of the plate deformation with a similar off-center load.

This example illustrates two capabilities of CoSolveEM. First, we can handle multiple non-zero voltage boundary conditions. Second, the solution for the mechanical deformation of the plate has no significant symmetry; the problem requires a full $3 \mathrm{D}$ solution in the mechanical domain.

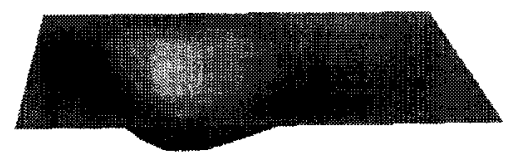

Figure 5 An exaggerated view of the plate deformation under a load of $s 1=40 \mathrm{~V}$ and $s 2=30 \mathrm{~V}$. This view looks along the $y$ axis and the electrodes line up on the left.

\section{Comb Finger Levitation}

Levitation [9] is an effect often encountered in comb-drive designs. It only appears in problems with more than two conductors, and its modeling 
usually requires going beyond a parallel plate approximation for the capacitance. It is also a coupled problem. Figure 6 shows a test model we use to explore levitation effects on structures like comb fingers. The model has three fingers, each 20 $\mathrm{x} 1 \mathrm{x} 1 \mu \mathrm{m}$ suspended $2 \mu \mathrm{m}$ over a ground plane. The central finger is fixed at its left end, and bends up under the levitation force. The outer two fingers are fixed.

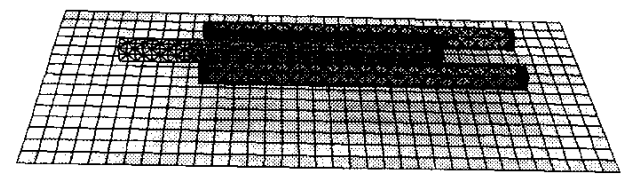

Figure 6 Comb fingers example. The example contains 3 fingers over a ground plane.

Tip Displacement

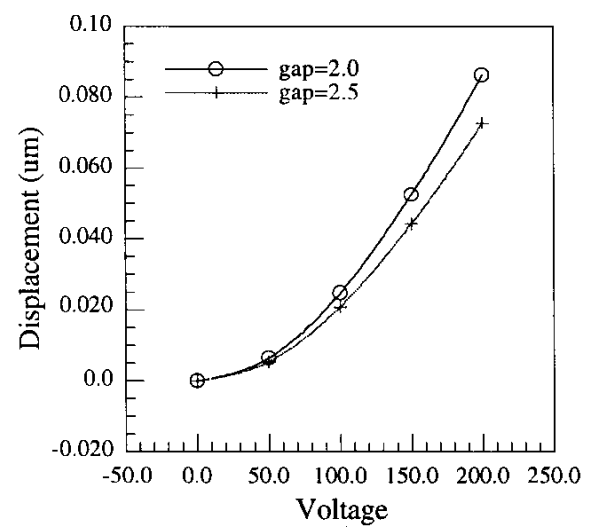

Figure 7 A graph from CoSolve-EM, showing the tip displacement vs. voltage for two different gap spacings to the ground plane.

Figure 7 shows the tip displacement vs. voltage curves for the tip of the central finger, when the voltage is applied to the two fixed fingers. We have run the CoSolve analysis for two different gaps between the fingers and the ground plane. Using the MemBase class library CoSolve-EM can vary the gap spacing itself, so the simulations for gaps of 2.5 $\mu \mathrm{m}$ and $2.0 \mu \mathrm{m}$ can be performed in the same run of CoSolve-EM, without rebuilding the model.
This example demonstrates two capabilities of CoSolve-EM. First, we can use the MemBase class library to apply translations to arbitrary parts of the model (the ground plane in this case) and second, we have used the SNGCR procedure for solving this model, since at high enough voltages all levitation problems begin to fail to converge under simple relaxation.

\section{Touch-Down Pressure Sensors}

Touch-down-mode capacitive pressure sensors provide an interesting example of devices in which it is important to estimate the capacitance of a deformed structure.

In this example we build a test model from a $150 \mu \mathrm{m}$ radius circular plate $10 \mu \mathrm{m}$ thick, $1 \mu \mathrm{m}$ above a ground plane. The rim of the circular plate is fixed in CoSolve-EM and a rigid surface $0.5 \mu \mathrm{m}$ above the ground plane and appropriate interface elements are created in CoSolve-EM. The device is meant to operate in a regime in which the pressure is high enough to cause the plate to touch down on the rigid surface.

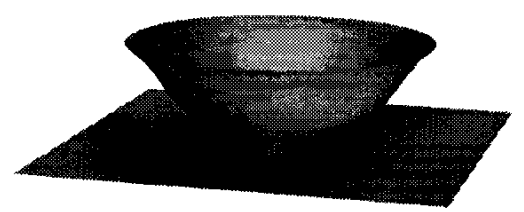

Figure 8 Image in CoSolve of the model, at 20 MPa. We show the bottom face of the plate and the ground plane. The $\mathrm{Z}$ scale is magnified by $100 \mathrm{x}$ for this visualization. The plate has radius $=150 \mu \mathrm{m}$, thickness $=10 \mu \mathrm{m}$, gap $=1 \mu \mathrm{m}$. The interface is 0.25 $\mu \mathrm{m}$ above the ground plane.

Figure 8 shows the bottom surface of the deformed plate. Figure 9 shows the capacitance vs. pressure and contact force vs. pressure for the range $0-20$ $\mathrm{MPa}$.

This example provides a demonstration of three features of CoSolve-EM. We can insert interface elements in CoSolve-EM (even if they were not present in the original meshed model) to solve contact problems; we can extract the reaction forces against those interfaces and the capacitance of the deformed structure; and we can vary any applied pressure in the problem to generate figures such as Figure 9. 


\section{Capacitance and Contact Force}

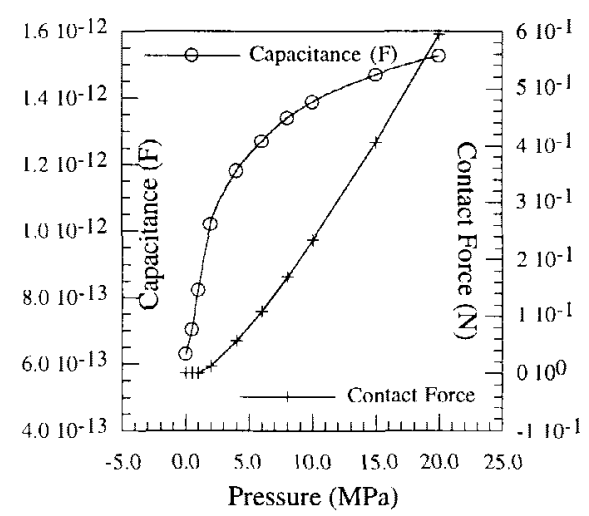

Figure 9 A graph from one CoSolve run showing the capacitance vs. pressure and the contact force vs. pressure for the touch down pressure sensor. Note that the contact force is 0 below $2 \mathrm{MPa}$, as the plate is not in contact at those pressures

\section{Curved Electrode Actuators}

Curved electrode actuators have been designed and built at Twente University and are discussed in more detail elscwhere [10]. Basically a flat beam is pulled in laterally against a curved electrode containing grounded "bumpers", both positioned above a ground plane. Such a device is shown in Figure 10. These devices have been built with various curvatures and various bumper locations. We model one example of such a device. In the model, $x$ is along the length of the beam, $y=-2.0$ is the plane of the ground plane, and the principal motion of the beam is along $z$, towards the fixed electrode. The beam is $515 \mu \mathrm{m}$ long, $5 \mu \mathrm{m}$ high and $2 \mu \mathrm{m}$ wide, made of polysilicon, with $\mathrm{E}=150 \mathrm{GPa}$. At $\mathrm{x}=0$ there is a gap of $3 \mu \mathrm{m}$ between the beam and the fixed electrode. The fixed electrode is $5 \mu \mathrm{m}$ high, $20 \mu \mathrm{m}$ wide, and extends $500 \mu \mathrm{m}$ along $x$ with curve:

$$
z=\frac{x^{3}}{500^{3}} 28
$$

Such a model is shown in Figure 11. In order to simulate a real device, we insert an interface (using CoSolve) $2 \mu \mathrm{m}$ away from the curved electrode and attach it to nodes every $50 \mu \mathrm{m}$ along the movable beam; this models bumpers every $50 \mu \mathrm{m}$ along the curved electrode.
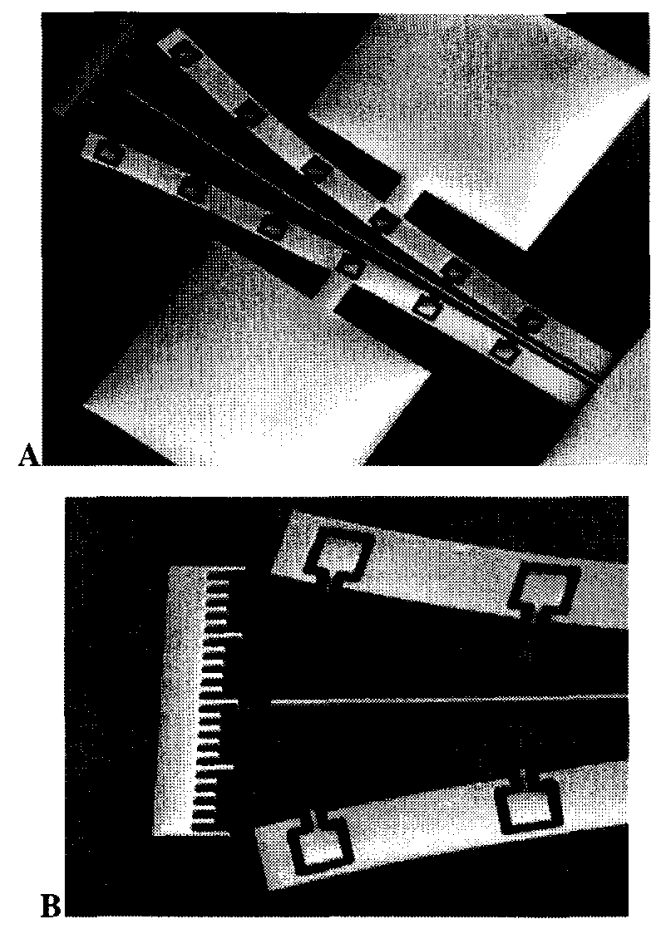

Figure 10 SEM photographs of Curved Electrode Actuators. A shows the entire curved electrode actuator. The thin central beam is movable, being fixed only on it rightmost extreme. In $\mathbf{B}$, a close-up of the tip region, the bumper structures are visible. The bumpers are $2 \mu \mathrm{m}$ wide and protrude $2 \mu \mathrm{m}$ from the curved electrode. They prevent the beam from touching the electrode and shorting out.

Figure 12 shows both measured data and the CoSolve-EM simulation of such a device for displacement of the beam at $x=500 \mu \mathrm{m}$ vs. applied voltage. This problem is a contact problem, and as mentioned above, CoSolve-EM must currently run contact problems in relaxation, not SNGCR.

However the curved electrode actuator is also a levitation problem, and at higher voltages $(>\sim 70 \mathrm{~V})$ it requires SNGCR to converge. Because of this difficulty we have suppressed levitation in this calculation by fixing the top of the movable beam to move only in $x$ and $z$. Notwithstanding that simplification, Figure 12 still shows good agreement between model and data out to $80 \mathrm{~V}$, and qualitative agreement everywhere. The model is systematically overestimating the force applied to the beam, as we would expect from the suppression of levitation. 


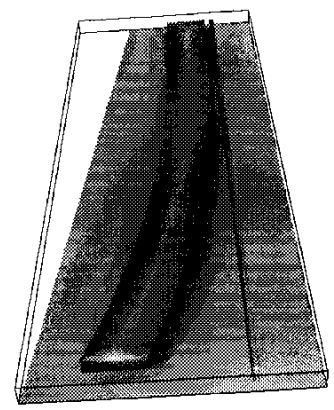

Figure 11 An image in CoSolve of beam, curved electrode and ground plane. The beam is $515 \times 5 \times 2 \mu \mathrm{m}$, the electrode is $500 \times 5 \times 20$ $\mu \mathrm{m}$ with curve $\mathrm{z}=28(\mathrm{x} / 500)^{3}$, both are $2 \mu \mathrm{m}$ above the ground plane.

Measured Data and Model

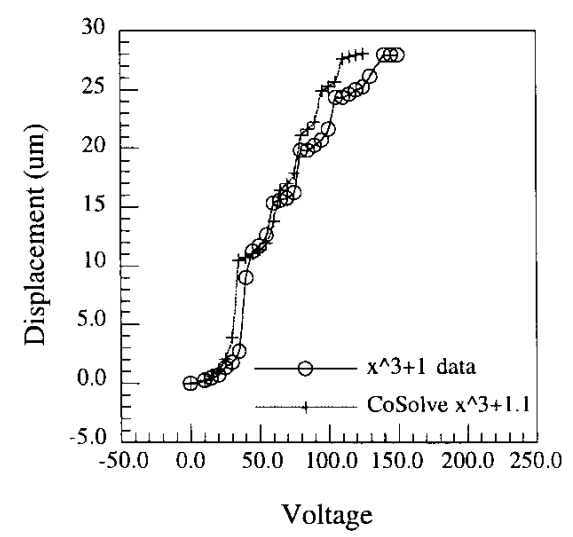

Figure 12 A graph showing measured data from a curved electrode actuator and a corresponding run of CoSolve-EM. The bumpers (every $50 \mu \mathrm{m}$ at $2 \mu \mathrm{m}$ away from the curved electrode) are added in CoSolve. This simulation is run with levitation suppressed.

\section{Conclusion}

We have described the envelope of problems that can now be attacked using CoSolve-EM. It includes problems of: pull-in, exact deformation, multiple conductors/voltages, levitation, surface contact, and various combinations of the above. We believe that CoSolve-EM is able to solve quasi-static coupled electro-mechanics for a range of problems that includes many which are interesting to MEMS designers.
Beta versions of CoSolve-EM are now running at MIT and at UC Berkeley. We intend a general release in 1995.

\section{Acknowledgments}

This work is supported by ARPA/ESTO under contract J-FBI-92-196. We would like to acknowledge the contributions of King Yu for work on the Universal File translator and Gregory Pal for work on the MemBase class library. We also acknowledge influential discussions with Jacob White, He Yie, Xuejun Cai and Peter Osterberg.

1 B.E.Artz and L.W.Cathey, "A Finite Element Method for Determining Structural Displacements Resulting from Electrostatic Forces", Proc. IEEE Solid State Sensor and Actuator Workshop, Hilton Head, SC, June 1992, pp. 190193

2 H.U. Schwarzenbach, J.G. Korvink,M. Roos, G. Sartoris, and E. Anderheggen, "A micro electro mechanical CAD extension for SESES", J. Micromech. Microeng. v3 (1993) pp. 118-122.

3 X. Cai, H. Yie, P. Osterberg, J. Gilbert, S. Senturia, and J. White, "A Relaxation/Multipole-Accelerated Scheme for Self-Consistent Electromechanical Analysis of Complex 3-D Microelectromechanical Structures", Proc. Int. Conf. on Computer-Aided Design, Santa Clara, CA, November 1993, pp. 270-274.

4 ABAQUS Manual, Hibbitt, Karlsson \& Sorenson, Inc. 1080 Main Street, Pawtucket, RI 02860, USA

5 K. Nabors and J. White, "FastCap: A multipoleaccelerated 3-D capacitance extraction program", IEEE Transactions on Computer-Aided Design, vol 10 no. 10 , Nov. 1991, pp. 1447-1459.

6 Software Development Group, Geometry Center, University of Minnesota, 1300 South Second Street, Suite 500, Minneapolis, MN 55454, USA, or see http://www.geom.umn.edu/welcome.html

7 John K. Ousterhout, "Tcl and the Tk Toolkit", Addison-Wesley ISBN 0-201-63337-X, 1994.

8 K. Najafi and K. Suzuki, "A Novel Technique and Structure for the Measurement of Intrinsic Stress and Young's Modulus of Thin Films", Proc. MEMS '89, Salt Lake City, Feb 1989, pp. 96-97. and P. Osterberg, H. Yie, X. Cai, J. White, and S. Senturia, "Self Consistent Simulation and Modeling of Electrostatically Deformed Diaphragms," Proc. MEMS ‘94, Oiso Japan, Jan. 1994, pp. 28-32.

9 W.C. Tang, M.G. Lim, and R.T. Howe, "Electrostatic comb drive levitation and control method," Journal of Microelectromechanical Systems, v1, 4, 1992, pp. 170-178. 10 R Legtenberg et.al. this volume. 